\title{
Prevalência de Excesso de Peso em Crianças e Adolescentes de um Projeto Esportivo em Maceió-AL
}

\section{Prevalence of Excess Weight in Children and Adolescents Participating of a Sports Project in Maceió, Al, Brazil}

\author{
YANA MARA QUEIROZ DA SILVA ${ }^{1}$ \\ MAJA KRAGULJAC ${ }^{2}$ \\ RODRIGO BARBOSAALBUQUERQUE ${ }^{3}$ \\ FABIANO DE SOUZA FONSECA ${ }^{4}$
}

\section{RESUMO}

Objetivo: O objetivo do estudo foi descrever a prevalência de excesso de peso em crianças e adolescentes de um projeto esportivo na cidade de Maceió - AL. Material e Métodos: A amostra foi composta por 381 crianças e adolescentes, de ambos os gêneros e com idade entre $7 \mathrm{e}$ 16 anos. Os participantes foram submetidos a medidas de massa corporal e estatura para cálculo do Índice de Massa Corporal (IMC). Os índices de excesso de peso (sobrepeso e obesidade) foram definidos segundo padrões préestabelecidos para idade e gênero. Foi realizada uma análise descritiva entre as variáveis independentes: idade, gênero e modalidade esportiva. Resultados: A prevalência de sobrepeso e obesidade na amostra foi $21,16 \%$ e $7,87 \%$, respectivamente, o que presume uma alta prevalência de excesso de peso nas criancas e adolescentes do projeto esportivo $(29,13 \%)$. Os resultados evidenciaram que as crianças apresentaram índices mais elevados em comparação aos adolescentes. A prevalência de excesso de peso nos meninos foi superior à observada nas meninas e maiores prevalências de excesso de peso foram detectadas nos praticantes de Futsal e Natação. Conclusão: Estes resultados sugerem a implantação de ações de prevenção e combate ao excesso de peso para proteção da saúde das crianças e adolescentes do projeto esportivo.

\section{DESCRITORES}

Sobrepeso. Obesidade. Criança. Adolescente.

\begin{abstract}
Objective: The aim of the study was to describe the prevalence of excess weight in children and adolescents participating of a sports project in the city of Maceió, AL, Brazil. Material and Methods: The sample consisted of 381 children and adolescents of both gender aged 7 to 16 years. Participants underwent weight and height measurements to have their Body Mass Index (BMI) calculated. The rates of excess weight (overweight and obesity) were classified considering the age and gender. Descriptive analysis was performed for the independent variables: age, gender and sport. Results: The prevalence of overweight and obesity in the sample was found to be $21.16 \%$ and $7.87 \%$, respectively, which was considered to be a high prevalence of excessive weight in the children and adolescents $(29.13 \%)$. The results showed that children had higher rates compared to adolescents. The prevalence of excessive weight in the boys was higher than that observed in the girls. In addition, higher excessive weight frequencies were detected in the practitioners of futsal and swimming. Conclusion: These results reveal the need for interventional actions for health protection of the children and adolescents of the sports project.
\end{abstract}

DESCRIPTORS

Overweight. Obesity. Child. Adolescent.

1 Educadora Física, Maceió/AL, Brasil.

2 Professora Mestra do Curso de Educação Física do Centro Universitário Cesmac, Maceió/AL, Brasil.

3 Professor Mestre do Curso de Educação Física do Centro Universitário Cesmac, Maceió/AL. Doutorando em Ciências do Esporte na Universidade Federal de Minas Gerais (UFMG), Belo Horizonte/MG, Brasil.

4 Professor Mestre do Curso de Educação Física do Centro Universitário Cesmac, Maceió/AL, Brasil. 
$\mathrm{A}$ obesidade é uma doença crônica caracterizada pelo acúmulo excessivo de gordura corporal em um nível capaz de comprometer a saúde ${ }^{1}$. O excesso de gordura corporal pode gerar inúmeras consequências à saúde, pois é considerado um fator de risco para doenças cardiovasculares, cerebrovasculares, hipertensão arterial, além de estar associado à incidência de morbimortalidades e diversos tipos de câncer ${ }^{2,3}$. Atualmente, o excesso de peso é considerado uma pandemia de caráter mundial, devido ao aumento crescente de sua prevalência tanto em países desenvolvidos quanto em países em desenvolvimento e por ocorrer em diferentes estratos sociais e faixas etárias ${ }^{4}$.

Ainda que o excesso de peso represente vários riscos à saúde em todas as faixas etárias, a sua ocorrência na infância e adolescência sugere uma preocupação ainda maior em virtude de as complicações decorrentes da obesidade estarem relacionadas ao tempo que o indivíduo se mantém obeso ${ }^{2}$. Logo, quanto mais cedo ocorre o seu aparecimento, maiores serão suas implicações e consequências à saúde. Além disso, o excesso de peso na infância e adolescência está associada à sua persistência na idade adulta ${ }^{5-7}$. Como a ocorrência da obesidade está diretamente relacionada aos hábitos de vida ${ }^{8-9}$, a incorporação de hábitos saudáveis ainda na infância se torna fundamental para prevenir sua manifestação em idades mais avançadas e evitar suas consequências à saúde. Como o excesso de peso na infância e adolescência representa vários riscos à saúde, o conhecimento sobre suas características epidemiológicas torna-se um importante mecanismo para a implantação de medidas de prevenção e proteção à saúde.

No Brasil, o excesso de peso na infância e adolescência, representado pelo sobrepeso e obesidade, apresenta índices de prevalência semelhantes aos identificados em países desenvolvidos ${ }^{10}$. Estudos realizados com pré-escolares norte-americanos ${ }^{11}$ e préescolares britânicos ${ }^{12}$ verificaram uma prevalência de sobrepeso e excesso de peso equivalente a $10,8 \%$ e $26,3 \%$, respectivamente. Resultados similares foram encontrados em estudos brasileiros realizados com crianças e adolescentes que apontam prevalências de excesso de peso que variam entre $10,6 \%$ e $33,7 \%^{14-19}$. Foi verificada uma prevalência de excesso de peso equivalente a $24,5 \%$ para o gênero feminino e $17,5 \%$ para o gênero masculino em crianças e adolescentes da cidade de Montes Claros - $\mathrm{MG}^{13}$. Em um estudo com toda a população de escolares entre 7 e 10 anos de idade na cidade de Santos -SP os resultados indicaram que $33,7 \%$ das crianças apresentavam excesso de peso $(15,7 \% \text { de sobrepeso e } 18,0 \% \text { de obesidade })^{14}$. Em um estudo de abrangência nacional com crianças menores de cinco anos, encontraram uma prevalência de excesso de peso equivalente a $10,6 \%{ }^{15}$. Os resultados acima apontam que o problema de excesso de peso tem sido constatado cada vez mais precocemente.

Embora no Brasil alguns estudos apontem que a prevalência de excesso de peso em crianças e adolescentes seja mais crescente entre as regiões Sul e Sudeste do país ${ }^{16}$, índices preocupantes também foi encontrado em crianças e adolescentes da região Nordeste. Foi identificado um percentual de $13,7 \%$ de excesso de peso em escolares de 5 a 10 anos de idade na cidade de Feira de Santana-BA (9,3\% de sobrepeso e $4,4 \%$ de obesidade $)^{17}$. Do mesmo modo, foi encontrada uma prevalência de $13,8 \%$ de excesso de peso em crianças e adolescentes com idades entre 7 e 17 anos na cidade de Maceió - AL, sendo 9,3\% de sobrepeso e de $4,5 \%$ de obesidade ${ }^{18}$. Em uma análise no período de 1980 a 2000, foram encontrados indicativos que apontam para uma tendência crescente de sobrepeso e obesidade em adolescentes do gênero masculino em todos os estados da região Nordeste ${ }^{19}$. Uma justificativa para esses resultados pode ser atribuída ao crescente processo de transição nutricional pelo qual o país vem passando, onde a região Nordeste, até meados da década de 80, apresentava elevados índices de subnutrição, atualmente apresenta índices alarmantes de excesso de peso em crianças e adolescentes.

Os estudos epidemiológicos com base populacional servem de ferramenta para que políticas públicas de saúde possam ser implantadas por órgãos governamentais (municipal, estadual ou federal) no intuito de modificar os quadros de excesso de peso apresentados na população infanto-juvenil ${ }^{20,21}$. Entretanto, as ações de proteção à saúde e prevenção do excesso de peso, advindas de políticas públicas, nem sempre são implantadas rapidamente. E ainda, muitas vezes não possuem a abrangência necessária para atingir a totalidade da população afetada. Uma alternativa para solucionar esses problemas é a implantação dessas ações diretamente pelas instituições sociais que atendem a crianças e adolescentes, tais como as escolas e projetos sociais. Medidas educacionais de prevenção e até mesmo programas de intervenção de combate à obesidade são exemplos de ações que têm demonstrado eficácia no combate ao excesso de peso na infância e adolescência ${ }^{21}$. Portanto, estudos descritivos que visam identificar o excesso de peso diretamente em instituições sociais que atendem crianças e adolescentes são importantes, pois além de complementar as informações oriundas de estudos epidemiológicos com base populacional, proporcionam a implantação de medidas pontuais e mais rápidas de 
prevenção e combate ao excesso de peso, capaz de abranger a totalidade da população afetada.

Tendo em vista que a condução de estudos descritivos em instituições sociais de cunho educativo que atendem a crianças e adolescentes pode auxiliar na implantação de medidas pontuais para prevenir o excesso de peso nessa população, o objetivo do presente estudo foi descrever a prevalência de excesso de peso em crianças e adolescentes participantes de um projeto esportivo na cidade de Maceió-AL.

\section{MATERIAL E MÉTODOS}

\section{População e Amostra}

Trata-se de um estudo do tipo descritivo transversal com abordagem quantitativa, realizado no ano de 2012. A amostra do estudo foi composta por 381 crianças e adolescentes, de ambos os gêneros, com faixa etária entre 7 a 16 anos, integrantes de um projeto esportivo na cidade de Maceió-AL. A amostra representou $73,5 \%$ da população de crianças e adolescentes participantes do projeto (total de 518 participantes), em que cada integrante participava de apenas uma modalidade esportiva dentre as quatro modalidades desenvolvidas: natação, handebol, futsal e voleibol. Fizeram parte da amostra somente as crianças e adolescentes que aceitaram o convite e apresentaram o "Termo de Consentimento Livre e Esclarecido" devidamente assinado pelo seu responsável.

\section{Instrumentos e Procedimentos}

Os participantes foram submetidos à avaliação antropométrica envolvendo medidas de massa corporal e estatura. Para a medida da massa corporal, os indivíduos foram avaliados descalços e usando roupas leves. A mesma foi aferida por uma balança eletrônica portátil da marca G-TECH fabricada na China (Zhongshan Camry Eletronic Ltda.), modelo GLASS 7, com precisão de $0,1 \mathrm{~kg}$ e capacidade máxima de $150 \mathrm{~kg}$. Durante a medição, o avaliador se posicionava em pé de frente para escala de medida, enquanto o avaliado subia na plataforma da balança cuidadosamente e se posicionava no centro da mesma, com ombros descontraídos e braços soltos lateralmente ${ }^{22}$.

A estatura foi determinada em um estadiômetro portátil Sanny Standard produzido no Brasil (American Medical do Brasil Ltda.). O estadiômetro possuía extensão de 2 metros, precisão de $0,1 \mathrm{~cm}$ e um esquadro embutido. Os indivíduos ficavam em posição ortostática, pés descalços e unidos, com as superfícies posteriores do calcanhar, cintura pélvica, cintura escapular e região occipital em contato com o estadiômetro. O esquadro, num ângulo de $90^{\circ}$ em relação à escala, tocava o ponto mais alto da cabeça no final de uma inspiração máxima, sendo neste momento realizada a leitura da medida ${ }^{22}$. A medida da estatura foi realizada uma única vez.

Os dados foram coletados durante o segundo semestre de 2012 e todos os procedimentos realizados neste estudo seguiram as normas da resolução 196/96 do Conselho Nacional de Saúde para pesquisas em seres humanos e foram aprovados pelo Comitê de Ética em Pesquisa institucional ( $\left.\mathrm{N}^{\mathrm{0}} 1312 / 12\right)$.

\section{Análise dos Dados}

O sobrepeso e a obesidade foram definidos a partir do cálculo do Índice de Massa Corporal (IMC) de acordo com a equação: IMC = massa corporal $/$

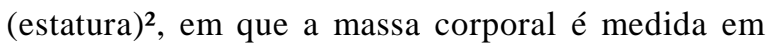
quilogramas $(\mathrm{kg})$ e a estatura em metros $(\mathrm{m})$.

Para a classificação do grau de excesso de peso (sobrepeso e obesidade) foram escolhidos pontos de corte para gênero e idade ${ }^{23}$, conforme a tabela 1. Para feito de análise dos dados, a idade dos indivíduos foi calculada em meses e obtida pela diferença entre a data da coleta de dados e a data de nascimento, sendo classificados como crianças os indivíduos de 7 a 11 anos e como adolescentes os indivíduos de 12 a 16 $\operatorname{anos}^{23}$.

O sobrepeso e a obesidade foram classificados considerando a idade e o gênero. Por exemplo, para a idade de 7 anos, foram considerados com sobrepeso aqueles sujeitos que apresentaram o IMC igual ou acima dos valores do limite para sobrepeso (IMC eŠ 17,92 kg/ $\mathrm{m}^{2}$ para o gênero masculino e e $\breve{S} 17,75$ para o gênero feminino) e abaixo do limite para obesidade. Foram considerados obesos aqueles sujeitos que apresentaram o IMC igual ou acima dos valores limite para obesidade (IMC eŠ $20,63 \mathrm{~kg} / \mathrm{m}^{2}$ para o gênero masculino e eŠ $20,51 \mathrm{~kg} / \mathrm{m}^{2}$ para o gênero feminino). $O$ excesso de peso foi definido pelo somatório dos índices de sobrepeso e obesidade (sobrepeso + obesidade). Esse procedimento foi realizado para todos os indivíduos com idades entre 7 e 16 anos, tanto do gênero masculino quanto do gênero feminino.

Para análise da prevalência de excesso de peso foi realizada uma estatística descritiva através do aplicativo Microsoft Excel versão 2010, que buscou apresentar e descrever os resultados representativos de cada variável (idade, gênero e modalidade esportiva) por meio de porcentagens. 


\begin{tabular}{|c|c|c|c|c|}
\hline \multirow{2}{*}{ Idade (anos) } & \multicolumn{2}{|c|}{ Sobrepeso } & \multicolumn{2}{|c|}{ Obesidade } \\
\hline & Masculino & Feminino & Masculino & Feminino \\
\hline 7 & 17,92 & 17,75 & 20,63 & 20,51 \\
\hline 7.5 & 18.16 & 18.03 & 21.09 & 21.01 \\
\hline 8 & 18,44 & 18,35 & 21,60 & 21,57 \\
\hline 8.5 & 18.76 & 18.69 & 22,17 & 22,18 \\
\hline 9 & 19,10 & 19,07 & 22,77 & 22,81 \\
\hline 9,5 & 19,46 & 19,45 & 23,39 & 23,46 \\
\hline 10 & 19.84 & 19.86 & 24.00 & 24.11 \\
\hline 10,5 & 20,20 & 20,29 & 24,57 & 24,77 \\
\hline 11 & 20.55 & 20.74 & 25.10 & 25.42 \\
\hline 11,5 & 20,89 & 21,20 & 25,58 & 26,05 \\
\hline 12 & 21,22 & 21,68 & 26,02 & 26,67 \\
\hline 12,5 & 21,56 & 22,14 & 26,02 & 26,67 \\
\hline 13 & 21,91 & 22,58 & 26,84 & 27,76 \\
\hline 13.5 & 22,27 & 22.98 & 27,25 & 28.20 \\
\hline 14 & 22,62 & 23,34 & 27,63 & 28,57 \\
\hline 14.5 & 22.96 & 23,66 & 27.98 & 28.87 \\
\hline 15 & 23,29 & 23,94 & 28,30 & 29,11 \\
\hline 15,5 & 23,60 & 24,17 & 28,60 & 29,29 \\
\hline 16 & 23.90 & 24.37 & 28.88 & 29.43 \\
\hline
\end{tabular}

Fonte: Cole et al. ${ }^{23}$

\section{RESULTADOS E DISCUSSÃO}

A amostra do estudo foi dividida por idade, gênero e modalidade esportiva praticada pelas crianças e adolescentes no projeto social (tabela 2). Foi possível observar um equilíbrio entre o número de crianças ( 7 a 11 anos) e adolescentes (12 a 16 anos) participantes do projeto esportivo, com frequências de $52,23 \%$ e $47,77 \%$, respectivamente. Porém, quando observada a variável gênero, notou-se que a maioria dos participantes é pertencente ao gênero masculino $(71,13 \%)$. Dentre as modalidades desenvolvidas no projeto, o Futsal e a Natação se mostraram com o maior número de participantes (48,03\% e 31,76\%, respectivamente), seguidas pelas modalidades Handebol e Voleibol (12,86\% e 7,35\%, respectivamente). O tempo médio de prática esportiva da amostra investigada foi de $2,2 \pm 1,3$ anos e todos os participantes praticavam suas respectivas modalidades esportivas de 2 a 3 sessões/semana, com duração de 60 minutos cada, totalizando um volume mínimo de 2 horas/semana e máximo de 3 horas/semana.

Os resultados globais referentes à prevalência de sobrepeso, obesidade e excesso de peso são apresentados na tabela 3. A prevalência total de excesso de peso da amostra foi de $29,13 \%$, sendo que houve uma maior prevalência de sobrepeso $(21,26 \%)$ em relação à obesidade $(7,87 \%)$ entre as crianças e adolescentes participantes do projeto esportivo. A prevalência total observada em 2012 foi superior à verificada nos estudos com população norte-americana $(25,6 \%)$ e maior que o dobro da média verificada no Brasil (13,6\%), conforme resultados oriundos de pesquisas de abran-gência mundial com crianças e adolescentes nos períodos de 1974-1975 e 1996-199724. Os resultados indicaram elevada prevalência de excesso de peso nas crianças e adolescentes participantes do projeto.

Foi verificado no presente estudo uma prevalência de excesso de peso superior ao dobro da frequência $(13,8 \%)$ encontrada em um estudo de base populacional em escolares com a mesma faixa etária em Maceió-AL ${ }^{18}$. Tais resultados sugerem que a prevalência de excesso de peso em crianças e adolescentes verificada em contextos específicos pode divergir de estudos de abrangência populacional em uma mesma localidade e apresentar índices ainda mais elevados de excesso de peso. Essas evidências reforçam a importância de estudos descritivos que buscam identificar o excesso de peso na infância e adolescência de forma localizada em contextos sociais específicos (escolas, projetos educacionais, projetos sociais), para que medidas de combate ao excesso de peso possam ser tomadas de forma pontual. 


\begin{tabular}{|c|c|c|}
\hline Variáveis & Total $-\mathbf{n}$ & Frequência - \% \\
\hline \multicolumn{3}{|l|}{ Idade } \\
\hline 7 a 11 anos & 199 & 52,23 \\
\hline $\begin{array}{l}12 \text { a } 16 \text { anos } \\
\text { Gênero }\end{array}$ & 182 & 47.77 \\
\hline Feminino & 110 & 28.87 \\
\hline Masculino & 271 & 71,13 \\
\hline \multicolumn{3}{|l|}{ Modalidade } \\
\hline Praticada & & \\
\hline Natação & 121 & 31,76 \\
\hline Handebol & 49 & 12,86 \\
\hline Futsal & 183 & 48,03 \\
\hline Voleibol & 28 & 7.35 \\
\hline
\end{tabular}

\begin{tabular}{ccc} 
Tabela 3. Prevalência de sobrepeso, obesidade e \\
excesso de peso encontrado na amostra. \\
\hline \multirow{3}{*}{ Variável } & \multicolumn{3}{c}{ Amostra } \\
& Total $-\mathrm{n}$ & Prevalência - \% \\
\hline Sobrepeso & 81 & 21,26 \\
Obesidade & 30 & 7,87 \\
Excesso de peso & 111 & 29,13 \\
\hline
\end{tabular}

\begin{tabular}{|c|c|c|c|c|c|c|c|}
\hline \multirow{3}{*}{ Variável } & & \multicolumn{6}{|c|}{ Classificacão } \\
\hline & & \multicolumn{2}{|c|}{ Sobrepeso } & \multicolumn{2}{|c|}{ Obesidade } & \multicolumn{2}{|c|}{$\begin{array}{c}\text { Excesso de } \\
\text { Peso }\end{array}$} \\
\hline & & $n$ & $\%$ & $n$ & $\%$ & $n$ & $\%$ \\
\hline Idade & $\begin{array}{c}7 \text { a } 11 \text { anos } \\
12 \text { a } 16 \text { anos }\end{array}$ & $\begin{array}{l}49 \\
32\end{array}$ & $\begin{array}{l}12,86 \\
8,40\end{array}$ & $\begin{array}{l}16 \\
14\end{array}$ & $\begin{array}{l}4,20 \\
3,67\end{array}$ & $\begin{array}{l}65 \\
46\end{array}$ & $\begin{array}{l}17,06 \\
12,07\end{array}$ \\
\hline \multirow{3}{*}{ Gênero } & Meninos & 51 & 13,39 & 22 & 5,77 & 73 & 19,16 \\
\hline & Meninas & 30 & 7,87 & 8 & 2,10 & 38 & 9,97 \\
\hline & Natação & 32 & 8,40 & 12 & 3,15 & 44 & 11,55 \\
\hline Modalidade & $\begin{array}{l}\text { Handebol } \\
\text { Futsal } \\
\text { Voleibol }\end{array}$ & $\begin{array}{c}12 \\
32 \\
5\end{array}$ & $\begin{array}{l}3,15 \\
8.40 \\
1,31\end{array}$ & $\begin{array}{c}1 \\
15 \\
2\end{array}$ & $\begin{array}{l}0,26 \\
3.94 \\
0,52\end{array}$ & $\begin{array}{r}13 \\
47 \\
7\end{array}$ & $\begin{array}{c}3,41 \\
12,34 \\
1,84\end{array}$ \\
\hline
\end{tabular}

Os resultados encontrados no presente estudo são consistentes com os indicativos que apontam para uma tendência crescente de excesso de peso entre crianças e adolescentes na região Nordeste ${ }^{17,27}$ Uma justificativa para os dados encontrados pode ser atribuída aos hábitos de vida adotados na sociedade moderna. Atualmente, há um envolvimento cada vez maior das crianças e adolescentes em atividades de baixo gasto energético ( $\mathrm{TV}$, computador, videogame) e um aumento do consumo calórico em lanches pouco saudáveis nos intervalos das três principais refeições do dia (por exemplo, salgados industrializados, refrigerantes, sucos artificiais, chocolates, entre outros $)^{29,30}$. Esses aspectos têm sido diretamente relacionados à crescente expansão da obesidade ${ }^{3,4} \mathrm{e}$ à predisposição da criança ou adolescente se tornarem adultos obesos ${ }^{9}$.

Os resultados do excesso de peso foram analisados considerando as variáveis idade, gênero e modalidade esportiva (tabela 4). A análise isolada destas variáveis permite o direcionamento das ações de prevenção e combate ao excesso de peso conforme a distribuição da sua incidência. Apesar da alta prevalência de excesso de peso ter sido observada tanto nas crianças ( 7 a 11 anos) quanto nos adolescentes (12 a 16 anos), os resultados mostraram que sua ocorrência 
foi maior entre as crianças $(17,06 \%)$. Os achados desta pesquisa estão de acordo com os resultados na literatura ${ }^{18}$ que também identificaram maior prevalência de excesso de peso entre crianças em relação a adolescentes na cidade de Maceió-AL, o que sugere que o problema tem se mostrado cada vez mais precoce. Estudos longitudinais têm demonstrado que a prevalência de excesso de peso ainda na infância pode resultar em um aumento do aparecimento e desenvolvimento de morbidades na idade jovem, repercutindo suas consequências na vida adulta ${ }^{5-7,13,28}$. Portanto, a identificação do problema feita precocemente pode resultar em medidas mais eficazes para proteção da saúde das crianças atendidas pelo projeto esportivo, com o objetivo de prevenir e evitar a instalação da obesidade em idades mais avançadas.

Uma vez que os resultados encontrados demonstram que o problema do excesso de peso entre os integrantes do projeto esportivo é generalizado, independente da sua faixa etária, firma-se a idéia de que ações de prevenção devem ser inseridas dentro do projeto e direcionadas para todas as faixas etárias. Todavia, o excesso de peso se mostrou mais crescente nas crianças, o que sugere que ações de combate sejam centradas nos indivíduos de 7 a 11 anos, pois a prevalência de excesso de peso já está alta e, portanto, já instalada. Sendo assim, além de atacar o problema pontualmente nessa faixa etária, as ações de combate podem evitar sua ocorrência na adolescência e na idade adulta. Programas de intervenções foram observados em estudos de tratamento da obesidade ${ }^{3}$ e vistos que as ações mais eficazes na prevenção do ganho de peso são aquelas centradas na reeducação alimentar junto à participação dos pais na escolha dos alimentos e no aumento da prática de exercícios físicos. Com isso, os resultados aqui encontrados sugerem que, como medidas de prevenção, além da prática esportiva, sejam adotados programas de reeducação alimentar com a inclusão da família no papel de mediadores.

Em relação ao gênero, tem-se observado um crescimento da prevalência de excesso de peso na população infanto-juvenil, tanto no gênero masculino quanto no feminino ${ }^{17,18}$. O número de casos diagnosticados neste estudo indica uma prevalência de excesso de peso superior para o gênero masculino $(19,16 \%)$, estando de acordo com os achados de outros autores que também apontam maior prevalência de sobrepeso e obesidade no gênero masculino ${ }^{21,27,28}$, independente dos critérios utilizados. Uma possível preocupação com a imagem corporal imposta pela sociedade moderna pode ser uma possível explicação para a menor prevalência de excesso de peso entre as meninas, principalmente entre as adolescentes ${ }^{27}$. No entanto, dentro da faixa etária estudada, essa diferença em relação ao gênero se torna irrelevante devido à fase de maturação dos indivíduos ${ }^{28}$. Com isso, as prevalências de excesso de peso encontradas, tanto entre os meninos quanto entre as meninas, se torna um alerta para que ações de intervenção no projeto esportivo sejam direcionadas à ambos os gêneros e fundamentadas em garantir um perfil nutricional adequado para esses indivíduos, tendo em vista que o sobrepeso e a obesidade são fortes preditores para o aumento de morbimortalidades e redução da qualidade de vida $^{3}$.

Outro importante resultado que pode ajudar a direcionar as medidas de combate ao excesso de peso entre os participantes do projeto foi verificado na variável modalidade esportiva praticada. Os resultados revelaram maiores prevalências de excesso de peso entre os praticantes de futsal e natação $(12,34 \%$ e $11,55 \%$, respectivamente). Uma possível justificativa para maiores prevalências nessas duas modalidades pode ser dada ao fato de a maioria dos adeptos pertencerem ao gênero masculino (maior número de meninos participantes). Independente disso, os resultados encontrados sugerem que as ações de combate ao excesso de peso podem ser direcionadas especialmente para as crianças e os adolescentes matriculados nessas duas modalidades (futsal e natação) e que as ações de prevenção e proteção à saúde podem ser direcionadas às crianças e aos adolescentes matriculados nas demais modalidades (handebol e voleibol).

Além disso, visto que as crianças e os adolescentes investigados neste estudo estejam diretamente envolvidos com a prática regular de atividade física nas diferentes modalidades esportivas, ainda é possível supor que o consumo calórico dessa população é mais alto que o gasto energético total ${ }^{28}$. Assim, os resultados aqui encontrados sugerem o aumento da frequência semanal das atividades propostas nas modalidades esportivas com o objetivo de aumentar o gasto energético e combater o excesso de peso das crianças e adolescentes atendidas pelo projeto esportivo. Os principais alvos para atacar esse problema em crianças e adolescentes: diminuição da ingestão calórica, prevenção do ganho excessivo de peso e aumento do gasto energético ${ }^{2}$. Desta forma, é importante que se comece a monitorar a evolução da massa corporal dessas crianças e adolescentes ao longo de sua passagem pelo projeto esportivo e que medidas educativas que incentivem a adoção de hábitos saudáveis relacionados aos aspectos supracitados sejam tomadas ${ }^{2}$.

Os quadros encontrados neste estudo reforçam a importância de se realizarem estudos descritivos em 
instituições educacionais, a fim de investigar a prevalência de excesso de peso e influenciar a adoção de programas que estimulem hábitos de vida mais saudáveis, incluindo uma reeducação alimentar junto à prática regular de exercícios físicos. Essas são algumas possíveis intervenções capazes de auxiliar na mudança do cenário entre as crianças e adolescentes integrantes do projeto esportivo.

Devido às características e o propósito do estudo, algumas limitações devem ser apontadas. A principal delas é a dificuldade de generalização dos resultados por não se tratar de um estudo de base populacional. Como o fenômeno foi investigado em uma população e contexto específico, há limitações em sua abrangência mesmo em cenários similares. Outra limitação apresentada foi a ausência de controle de variáveis que exercem influência no IMC, como é o caso da maturação sexual, uma vez que o fenômeno da puberdade pode ocorrer de forma diferenciada entre indivíduos de mesma faixa etária nos indicadores da composição corporal ${ }^{21}$. Outra limitação que deve ser levada em consideração é o fato da medida da estatura ter sido realizada uma única vez, o que limita o grau de

\section{REFERÊNCIAS}

1. World Health Organization. Obesity: preventing and managing the global epidemic. Report of a Who Consultation group on obesity. Geneva; 1997. Disponível em: http://libdoc.who.int/trs/WHO_TRS_894.pdf. Acesso em: 30 set 2013.

2. Mello ED, Luft VC, Meyer F. Obesidade infantil: como podemos ser eficazes? J Pediatr. 2004; 80(3): 173182.

3. Sichieri R, Souza RA. Estratégias para prevenção da obesidade em crianças e adolescentes. Cad Saúde Pública. 2008; 24(2): S209-S234.

4. Oliveira $C L$, Fisberg M. Obesidade na infância e adolescência: uma verdadeira epidemia. Arq Bras Endocrinol Matab. 2003; 47(2): 107-108.

5. Freedman DS, Khan LK, Dietz WH, Srinivasan SR, Berenson, GS. Relationship of childhood obesity to coronary heart disease risk factors in adulthood: The Bogalusa heart study. J Pediatr. 2001; 3(108): 712718.

6. Laitinen J, Power C, Järvelin MR. Family social class, maternal body mass index, childhood body mass index, and age at menarche as predictors of adult obesity. Am J Clin Nutr. 2001; 74(3): 287-294.

7. Giugliano R, Carneiro EC. Fatores associados à obesidade em escolares. J Pediatr. 2004; 80(1): 1722.

8. Daniels SR. Cardiovascular disease risk factors and atherosclerosis in children and adolescents. Atherosclerosis. 2001; 3 (6): 469-485. consistência dos resultados, apesar de todos os avaliadores terem passado por um processo de treinamento previamente à coleta de dados.

\section{CONCLUSÕES}

Foram encontradas elevadas prevalências de excesso de peso em toda amostra investigada, especialmente nas crianças com idade entre 7 e 11 anos, do gênero masculino e praticantes das modalidades Futsal e Natação.

Devido aos problemas e complicações relacionados ao excesso de peso infantil e juvenil, os resultados aqui encontrados são preocupantes e sugerem a necessidade de implantação de ações de prevenção e proteção à saúde das crianças e adolescentes que integram o projeto esportivo. Neste sentido, torna-se relevante que o projeto insira ações de reeducação nutricional combinados com uma maior frequência da prática esportiva no intuito de combater o excesso de peso entre as crianças e adolescentes matriculadas.

9. Magarey AM, Daniels LA, Boulton TJ. Prevalence of overweight and obesity in Australian children and adolescents: reassessment of 1985 and 1995 data against new standard international definitions. Med $J$ Aust. 2001; 174(11): 561-564.

10. Silva GAP, Balaban G, Freitas MMV, Baracho JDS, Nascimento EMM. Prevalência de sobrepeso e obesidade em crianças pré-adolescentes matriculadas em escolas particulares de Recife, Pernambuco. Rev Bras Saúde Infantil. 2003; 3(3): 323-327.

11. Ogden CL, Troiano RP, Briefel RR, Kuczmarski RJ, Flegal $\mathrm{KM}$, Johnson CL. Prevalence of overweigth among preschool children in the United States, 1971 through 1994. In: Silva GAP, Balaban G, Freitas MMV, Baracho JDS, Nascimento EMM. Prevalência de sobrepeso e obesidade em crianças pré-adolescentes matriculadas em escolas particulares de Recife, Pernambuco. Rev Bras Saúde Infantil. 2003; 3(3): 323-327.

12. Bundred P, Kitchiner D, Buchan I. Prevalence of overweight and obese children between 1989 and 1998 : population based series of cross sectional studies. In: Silva GAP, Balaban G, Freitas MMV, Baracho JDS Nascimento EMM. Prevalência de sobrepeso e obesidade em crianças pré-adolescentes matriculadas em escolas particulares de Recife, Pernambuco. Rev Bras Saúde Infantil. 2003; 3(3): 323-327. 
13. Guedes DP, Neto JTM, Almeida MJ, Martins, Silva AJR. Impacto de fatores sociodemográficos e comportamentais na prevalência de sobrepeso e obesidade de escolares. Rev Bras Cineantropom Des Hum. 2010; 12(4): 221-231.

14. Costa RF, Cintra IP, Fisberg M. Prevalência de sobrepeso e obesidade em escolares da cidade de Santos, SP. Arq Bras Endocrinol Metab. 2006; 50: 6067.

15. Monteiro CA, Mondini L, Souza ALM, Popkin BN. Da desnutrição para a obesidade: a transição nutricional no Brasil. In: Silva GAP, Balaban G, Freitas MMV, Baracho JDS, Nascimento EMM. Prevalência de sobrepeso e obesidade em crianças pré-adolescentes matriculadas em escolas particulares de Recife, Pernambuco. Rev Bras Saúde Infantil. 2003; 3(3): 323-327.

16. Araújo EDS, Petroski EL. Estado nutricional e adiposidade de escolares de diferentes cidades brasileiras. Rev Educ Fís. 2002; 13(2): 47-53.

17. Oliveira AMA, Cerqueira EMM, Souza JS, Oliveira AC. Sobrepeso e obesidade infantil: influência dos fatores biológicos e ambientais em Feira de Santana, BA. Arq Bras Endocrinol Metab. 2003; 47(2): 144-150.

18. Mendonça MRT, Silva MAM, Rivera IR, Moura AA. Prevalência de sobrepeso e obesidade em crianças e adolescentes da cidade de Maceió. Rev Assoc Med Bras. 2010; 56(2): 192-196

19. Vasconcelo VL, Silva GAP. Prevalências de sobrepeso e obesidade em adolescentes masculinos, no nordeste do Brasil, 1980-2000. Cad Saúde Pública. 2003; 19(5): 1445-1451.

20. Pinheiro ARO, Freitas SFT, Corso ACT. Uma abordagem epidemiológica da obesidade. Rev Nutr. 2004; 17(4): 523-533.

21. Fernandes RA, Kawaguti SS, Agostini L, Oliveira AR, Ronque VER, Freitas Júnior IF. Prevalência de sobrepeso e obesidade em alunos de escolas privadas do município de Presidente Prudente - SP. Rev Bras Cineantropom Des Hum. 2007; 9(1): 21-27.

22. Beck CC, Diniz IMS, Gomes MA, Petroski EL. Ficha antropométrica na escola: o que medir e para que medir? Rev Bras Cineantropom Des Hum. 2007; 9(1): 107114.
23. Cole TJ, Bellizzi MC, Flegal KM, Dietz WH. Establishing a standard definition for child overweight and obesity worldwide: international survey. Br Med J. 2000; 320: 1240-1243.

24. Wang Y, Monteiro C, Popkin BM. Trends of obesity and underweight in older children and adolescents in the United States, Brazil, China, and Russia. Am J Clin Nutr. 2002; 75 (6): 971-977.

25. Monteiro CA, Conde WLA. Tendência secular da obesidade segundo estratos sociais: Nordeste e Sudeste do Brasil, 1975-1989-1997. Arq Bras Endocrinol Metab. 1999; 43:186-194.

26. Soar C, Vasconcelos FAG, Assis MAA, Grosseman S, Luna MEP. Prevalência de sobrepeso e obesidade em escolares de uma escola pública de Florianópolis, Santa Catarina. Rev Bras Saúde Matern Infantil. 2004; 4(4): 391-397.

27. Balaban G, Silva GAP. Prevalência de sobrepeso e obesidade em crianças e adolescentes de uma escola da rede privada de Recife. J Pediatr. 2001; 77(2): 96100.

28. Xavier MM, Xavier RM, Magalhães FO, Nunes AA, Santos VM. Fatores associados à prevalência de obesidade infantil em escolares. RBM. 2006; 40:105-108.

29. Barreto ACNG, Brasil LMP, Maranhão HS. Sobrepeso: uma nova realidade no estado nutricional de préescolares de Natal, RN. Rev Assoc Med Bras. 2007; 53(4): 311-316.

30. Bismarck-Nasr EM, Frutuoso MFP, Gambardella AMD. Importância nutricional dos lanches na dieta de adolescentes urbanos de classe média. Rev Paul Pediatr. 2006; 28(1):26-32.

\section{Correspondência}

Fabiano de Souza Fonseca

Rua Durval Guimarães 184, Apto 105 Bairro Ponta Verde Maceió - Alagoas - Brasil

CEP: 57035-060

E-mail: fabianoef@gmail.com 